\title{
Políticas de desarrollo económico local en las municipalidades de Chile: más allá del asistencialismo
}

\author{
Felipe Correa y Marco Dini
}

\section{Resumen}

¿En qué medida las políticas de desarrollo económico local se circunscriben en Chile a una concepción asistencialista? La hipótesis que se desarrolla en este trabajo se relaciona con que, vistas en conjunto, estas políticas sobrepasan ampliamente este paradigma. Para ejecutar el estudio se relevó una serie de 40 categorías de políticas mediante la lectura y sistematización de las políticas de desarrollo económico local propuestas por las municipalidades de Chile a través de 69 Planes de Desarrollo Comunal (PLADECO). En promedio las municipalidades proponen acciones en torno a 16 de las 40 categorías, pero son estas en conjunto las que pueden abarcar de forma satisfactoria los diversos desafíos del desarrollo económico local que cada municipalidad enfrenta de acuerdo a la realidad productiva comunal.

\section{Palabras clave}

Desarrollo económico, desarrollo local, política de desarrollo, planes de desarrollo, empleo, recursos humanos, productividad, desarrollo de empresas, indicadores del desarrollo, gobierno municipal, Chile

\section{Clasificación JEL}

$\mathrm{H} 75, \mathrm{O} 18, \mathrm{R} 58$

\section{Autores}

Felipe Correa es Asistente de Investigación de la División de Desarrollo Productivo y Empresarial de la Comisión Económica para América Latina y el Caribe (CEPAL). Correo electrónico: felipe.correa@un.org.

Marco Dini es Oficial de Asuntos Económicos de la División de Desarrollo Productivo y Empresarial de la Comisión Económica para América Latina y el Caribe (CEPAL). Correo electrónico: marco.dini@un.org. 


\section{Introducción}

La relevancia del enfoque local del desarrollo económico ha sido enfatizada por una vasta literatura, que además ha contribuido a destacar la importancia de las dinámicas territoriales en la generación y difusión de conocimientos, especialmente de los adaptativos y tácitos que surgen de la interacción entre los integrantes de los sistemas productivos locales. Asimismo, dicha literatura subraya el aporte de la cohesión social en cuanto a estimular la difusión de buenas prácticas y del aprendizaje compartido que se refiere a potenciar la capacidad de adaptación y la resiliencia frente a turbulencias externas y a la generación de aquel capital social de confianza recíproca que está en la base de los modelos de organización externa del trabajo que promueven la especialización productiva de las empresas (especialmente las de menor tamaño) y la complementación entre firmas independientes (Alburquerque y Dini, 2008; Alburquerque, 2004 y 2013; Becattini, 2002; Bianchi y Labory, 2014; Camuffo y Grandinetti, 2005).

A este elemento disciplinario se suma la importancia política, ejemplificada por las declaraciones emanadas de la Conferencia de las Naciones Unidas sobre los Asentamientos Humanos (Hábitat II, celebrada en 1996) y la reciente Conferencia de las Naciones Unidas sobre la Vivienda y el Desarrollo Urbano Sostenible (Hábitat III, de 2016). El documento final de Hábitat III otorga una función clave al concepto de desarrollo económico local pues en él se equiparan las instituciones locales a las que operan a nivel nacional o regional y se manifiesta el propósito de los gobiernos firmantes de actuar en pos del fortalecimiento de las instituciones de desarrollo locales (Naciones Unidas, 2016).

En todo el mundo, los gobiernos locales son actores clave para el diseño y la puesta en marcha de políticas de desarrollo económico y productivo. Su conocimiento de la realidad local y, en particular, de las necesidades, intereses y potencialidades que caracterizan y motivan a los actores locales les otorga herramientas importantes para el diseño de programas que resulten más pertinentes y eficaces. Al mismo tiempo, su cercanía con los integrantes de la comunidad les ofrece mayores oportunidades para generar espacios de diálogo y participación efectiva que permitan activar los recursos humanos y materiales de la localidad y volcarlos al diseño, puesta en marcha y revisión continua de las actividades de desarrollo económico.

Para entender cómo y en qué grado los gobiernos locales han llevado a la práctica estos aspectos que los colocan en un rol protagónico dentro del proceso de desarrollo económico, es imprescindible analizar el avance de las políticas planteadas por estos actores. En la medida que se cuente con más y mejor información, este análisis debería volverse cada vez más frecuente.

Sin embargo, a pesar del reconocimiento de que la contribución de los gobiernos locales a la definición de las políticas de desarrollo es igualmente importante que aquella de las instituciones nacionales o regionales (Naciones Unidas, 2016), este tema ha concentrado niveles de reflexión y estudio ciertamente escasos de parte de la disciplina económica, al menos en lo que respecta a América Latina, y en particular a Chile. Quizás por el carácter unitario del Estado, en Chile - a diferencia de otros Estados latinoamericanos donde la organización federal concede una importancia relativa mayor al aspecto local de las políticas de desarrollo - las municipalidades han sido consideradas históricamente como "el pariente pobre del Estado" (Salazar, 2013).

El presente estudio contribuye a la necesaria reflexión - hoy casi ausente- sobre las políticas de desarrollo económico local en Chile. A pesar de que cierta literatura revela que se han estudiado y sintetizado de forma cualitativa diversas políticas y experiencias de desarrollo económico local en América Latina (Alburquerque, 2004), este no ha sido el caso para Chile, siendo reconocida la escasez de estudios sobre el tema (Bravo, 2012).

Siguiendo un método de revisión de los Planes de Desarrollo Comunal (PLADECO) de 69 de las comunas más importantes del país, se logró identificar un conjunto de 40 categorías de acción 
de la política de desarrollo económico local, las cuales involucran una amplia gama de aspectos relacionados con el tema. De esta forma, el presente análisis pretende ser el primer intento de estudiar en forma sistemática este tipo de políticas en Chile.

Las políticas de desarrollo económico local analizadas dan cuenta, además, de experiencias novedosas en comunas específicas, que van más allá del enfoque tradicional de las políticas de desarrollo productivo comúnmente implementadas por los gobiernos locales. Las políticas tradicionales, circunscritas a la capacitación, la intermediación laboral y la entrega de información sobre programas de fomento productivo del gobierno central, se enmarcan dentro de lo que para varios autores es una orientación principalmente asistencialista (Alayón, 1991; Ziccardi, 2004; Artigas, 2005; Illanes, 2010), referida a una filosofía respecto de las políticas públicas que concibe a los agentes productivos más como objetos de beneficencia de estos programas - en un marco de receptividad pasiva - que como sujetos activos. De esta manera, la pregunta que motiva el presente estudio se relaciona con el grado en que las políticas de desarrollo económico local chilenas se circunscriben a este enfoque asistencialista. En ese sentido, el conocimiento aislado de diversas iniciativas de desarrollo económico local de Chile nos mueve a plantear la hipótesis de que efectivamente las municipalidades del país, consideradas como un grupo, sobrepasan ampliamente un marco asistencialista en lo que respecta a la política de desarrollo económico local.

Los resultados de la investigación sugieren que una cantidad significativa de municipalidades ha sobrepasado el marco tradicional de las acciones regidas por la concepción asistencialista. Cada municipalidad lo ha hecho de forma sui géneris, obligada quizás por la presión que implica el entender que el desarrollo económico es responsabilidad tanto del gobierno central como del gobierno local.

No obstante, las políticas de desarrollo económico que superan el marco tradicional no dejan de lado el componente asistencial - que representa el punto de partida -, sino que, al ampliarse su horizonte de responsabilidad, la acción de asistencia queda inserta en una estrategia de desarrollo más articulada. Los planes de desarrollo económico con participación ciudadana, las actividades productivas asentadas directamente en los organismos del gobierno local y los esquemas institucionales de coordinación público-privada son ejemplos de esta transformación.

En lo que sigue, el texto se organiza de la siguiente manera. La segunda sección contiene una descripción de las fuentes de información utilizadas y el método aplicado. En la tercera se describen las políticas de desarrollo económico local que fue posible relevar, ordenadas conforme a las 40 categorías propuestas, y se incluye una valoración de cada una de ellas y algunos elementos que podrían servir de modelo para la elaboración de políticas. La cuarta sección presenta una reflexión sobre las características de las comunas más y menos activas, con el fin de identificar de forma incipiente los factores que determinan el dinamismo en relación con las políticas. En la quinta y última sección se concluye sintetizando los principales hallazgos, junto con proponer futuras líneas de investigación.

\section{Método y fuentes de información}

Según la Ley Orgánica Constitucional de Municipalidades, es función privativa de este nivel de gobierno el "elaborar, aprobar y modificar el plan comunal de desarrollo" (art. 3), lo que se relaciona con la atribución esencial de "ejecutar el plan comunal de desarrollo y los programas necesarios para su cumplimiento" (art. 5), de modo que este plan constituye uno de los cuatro instrumentos mínimos de la gestión municipal (art. 6) ${ }^{1}$. De acuerdo con esta ley, "el plan comunal de desarrollo, instrumento rector del desarrollo en la comuna, contemplará las acciones orientadas a satisfacer las necesidades

1 Junto con el Plan Regulador Comunal, el Presupuesto Municipal Anual y la Política de Recursos Humanos. 
de la comunidad local y a promover su avance social, económico y cultural" (art. 7), es decir, se reconoce que el plan de desarrollo es quizás el elemento más importante de la política local.

Entre los estudios que se han realizado utilizando información de Planes de Desarrollo Comunal² se cuentan los de Montecinos (2006), Arredondo, Toro y Olea (2007), Valenzuela (2007), Olea (2011), Ruz y otros (2014) y Orellana, Mena y Montes (2016). Sin embargo, como ocurre en la mayoría de los casos mencionados, el estudio se ha centrado en el análisis de los procesos. Es decir, los autores evalúan las características formales del proceso de elaboración de los PLADECO, como la participación ciudadana, los niveles de transparencia y rendición de cuentas y, en general, los aspectos formales de la gobernanza y la institucionalidad. Este enfoque, a pesar de que permite relevar elementos esenciales del proceso de planificación local, no considera necesariamente la dimensión del contenido presente en los planes, aspecto que puede eventualmente resultar provechoso para el estudio comparado de las políticas.

Como parte del presente trabajo, se llevó a cabo una lectura individual y completa de cada PLADECO para elaborar las estadísticas sobre frecuencia de cada categoría de políticas, prescindiendo de esta manera de programas computacionales por considerarse que la complejidad de la clasificación requiere, al menos en una etapa inicial, de una interpretación no mecánica de los textos.

En 2017, el país contaba con 15 regiones desagregadas en 54 provincias, las cuales a su vez estaban compuestas de 346 comunas y 345 municipalidades $^{3}$. En cuanto a las comunas, según el censo de población de 2012, 52 de ellas tienen más de 100.000 habitantes, y otras 107, entre 20.000 y 100.000 habitantes. La elección de las comunas en que se estudiaron los Planes de Desarrollo Comunal se basó en el criterio de la Sociedad Administradora de Fondos de Cesantía de Chile (AFC Chile) $)^{4}$, que inició sus operaciones en 2013 con sucursales propias ubicadas en comunas que cumplían alguno de los siguientes requisitos: i) ser capital regional; ii) ser capital provincial, o iii) tener más de 100.000 habitantes. Asimismo, se descartaron las capitales provinciales con menos de 20.000 habitantes $^{5}$. De este modo, el presente estudio refleja de manera preferente las particularidades y necesidades de las comunas grandes y mayoritariamente urbanas.

De los 69 Planes de Desarrollo Comunal analizados, 55 se encontraban vigentes en la fecha en que se hizo el estudio - lo que equivale a un $80 \%$ - y la mayoría de las municipalidades en que este no era el caso estaban trabajando en su actualización. Conforme a la Ley Orgánica Constitucional de Municipalidades, la vigencia mínima del Plan de Desarrollo Comunal es de cuatro años, y no se establece un plazo máximo (art. 7). Según la información proporcionada por la muestra de 69 planes, estos tienen una duración de 4,6 años en promedio, con una dispersión considerable y una correlación positiva entre el tamaño de la comuna y el horizonte de planificación: mientras las comunas con un mayor número de habitantes tienen un horizonte de planificación más largo, las comunas más pequeñas se acercan al mínimo legal de cuatro $a_{n ̃ o s}^{6}$ (véase el gráfico 1).

2 La ley se refiere a este instrumento como "plan comunal de desarrollo", pero en el ámbito de la política se utiliza con mayor frecuencia la denominación de Plan de Desarrollo Comunal, con su acrónimo PLADECO. En este estudio usamos la denominación de la política en vez de la legal.

3 La Municipalidad de Cabo de Hornos administra la agrupación de las comunas de Cabo de Hornos y Antártica.

4 AFC Chile administra los fondos de cesantía y, por ende, tiene vinculación directa con algunas municipalidades a través de los órganos locales de fomento productivo e intermediación laboral (como las Oficinas Municipales de Intermediación Laboral (OMIL)), quienes certifican la cesantía en el caso de que se cobre el seguro a través del Fondo de Cesantía Solidario. Esto asegura que en estas municipalidades existe un mínimo de institucionalidad y de políticas orientadas al empleo, la intermediación laboral, la capacitación y el fomento productivo.

5 Los estudios previos en que se analizan Planes de Desarrollo Comunal y se incluye un mayor número de comunas son los de Ruz y otros (2014), que abarca 30 comunas, y Orellana, Mena y Montes (2016), que comprende 58.

6 Las siguientes comunas tienen un horizonte de planificación de nueve años: Puente Alto, Antofagasta, Viña del Mar, San Bernardo y Curicó. 


\section{Gráfico 1}

Chile: horizonte de planificación del Plan de Desarrollo Comunal en relación con la cantidad de habitantes por comuna

(En número de años y número de personas)

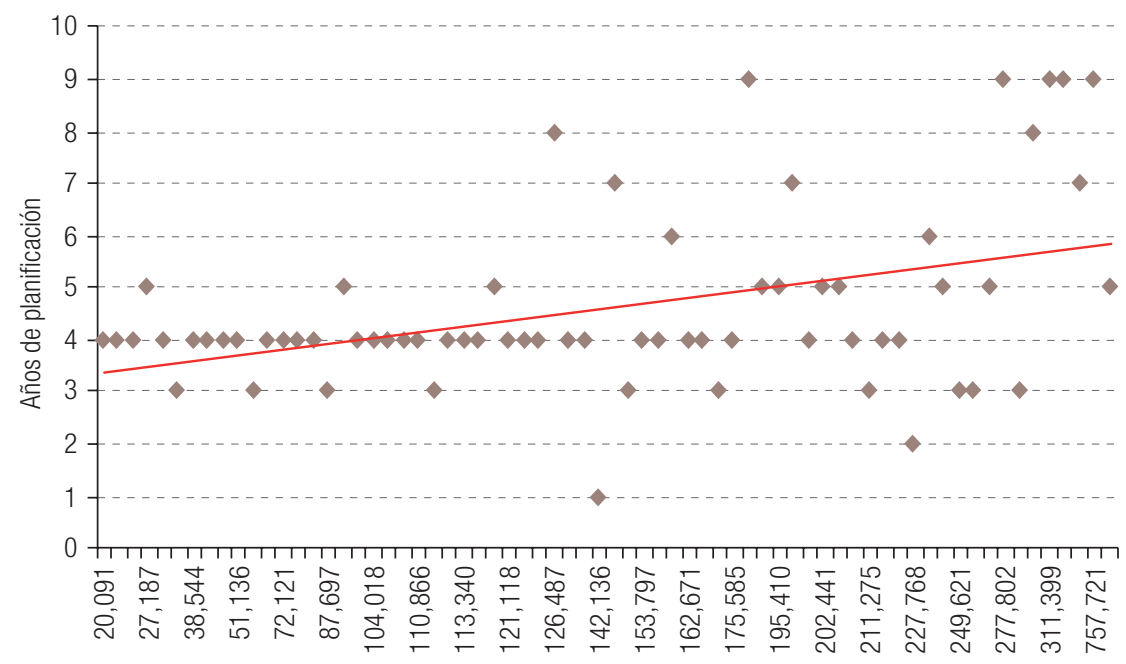

Población por comuna

Fuente: Elaboración propia, sobre la base de Planes de Desarrollo Comunal.

Otro elemento que considerar es el proceso de elaboración de los Planes de Desarrollo Comunal. De acuerdo con datos de la Dirección de Compras y Contratación Pública (ChileCompra), entre 2009 y 2015 se adjudicaron un total de 214 licitaciones relacionadas con la actualización de estos planes (véase el gráfico 2). Solo en 2015 el número de estas licitaciones ascendió a 48, lo que muestra que muchas municipalidades están externalizando esta función. A partir del cruce de la información contenida en la muestra utilizada y la que proviene de ChileCompra, es posible constatar que al menos el 58\% de los planes consultados fue elaborado por empresas consultoras externas.

\section{Gráfico 2}

Chile: licitaciones relacionadas con la elaboración y actualización de Planes de Desarrollo Comunal, 2009-2015

(En número de licitaciones)

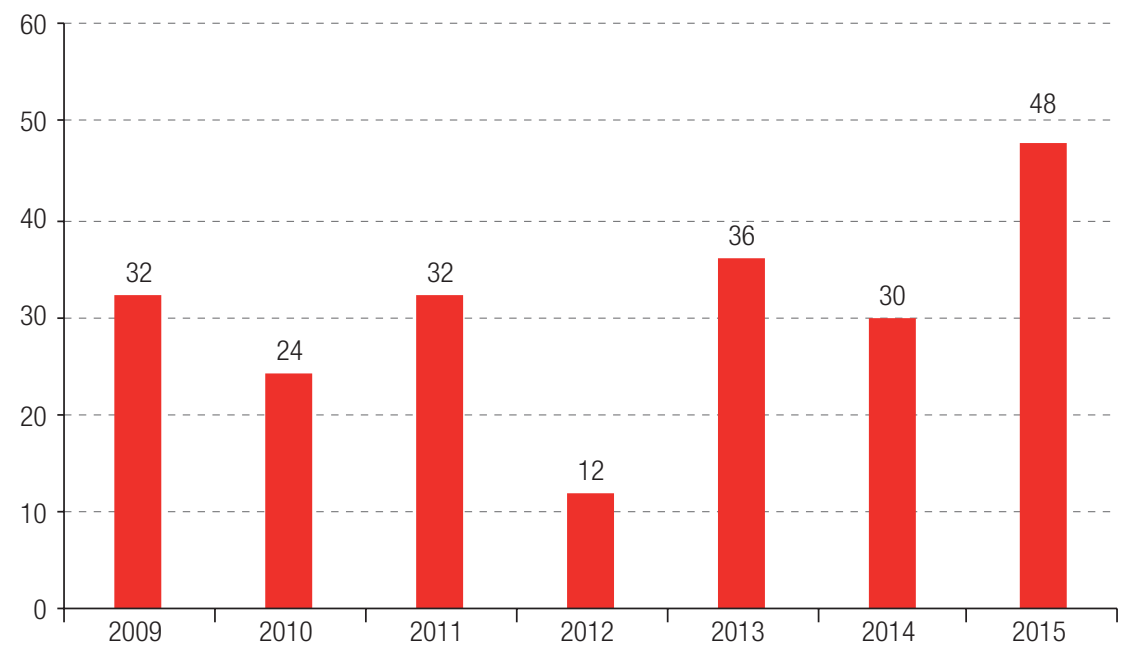

Fuente: Elaboración propia, sobre la base de información proporcionada por la Dirección de Compras y Contratación Pública (ChileCompra) del Ministerio de Hacienda. 
La escasez de personal con las competencias necesarias para desarrollar esta labor seguramente explica en parte este panorama. No obstante, la decisión de delegar esta función en una entidad externa a la municipalidad puede limitar seriamente la efectividad de la propuesta y debilitar el proceso de creación de capacidades autónomas en la municipalidad. El proceso de interpretación de la realidad económica y social, si se lleva a cabo en directo contacto con los actores locales, no solo es un ejercicio de recolección de información, sino también un momento clave para la construcción de relaciones de confianza entre la municipalidad y los miembros de la comunidad respectiva, lo que es vital para que las propuestas que surjan de este proceso obtengan el respaldo y la legitimidad necesarios para su implementación, tanto por parte del gobierno comunal como de la ciudadanía.

\section{Catastro de políticas de desarrollo económico local}

Cabe mencionar que las secciones de los PLADECO que abordan el desarrollo económico-productivo incorporan en algunos casos planes, programas o actividades que no tienen vinculación directa con esa temática. Para efectos del análisis, hemos descartado esas iniciativas. Al mismo tiempo, algunas medidas tienen que ver con el desarrollo económico-productivo, pero están dispersas en diferentes secciones de cada plan de desarrollo, como las de educación, ruralidad, temas indígenas, medioambiente y urbanismo. También prescindimos de estas propuestas para mostrar solo lo que en cada PLADECO se considera como parte de la estrategia de desarrollo económico-productivo, que constituye una especie de autodefinición de la política de desarrollo económico. Sin embargo, como se verá a continuación, el desarrollo económico-productivo se interseca con una gran cantidad de otros aspectos no esencialmente económicos, lo que es necesario tener en cuenta para que surja una visión sistémica sobre el proceso de desarrollo y para la coordinación entre los distintos agentes públicos y privados que actúan sobre el territorio.

Otro elemento al que es necesario referirse es que en el caso de que un plan, un programa o una actividad tengan relación con dos o más categorías, se incluyen en todas ellas para efectos de la elaboración de las estadísticas. El gráfico 3 muestra las 40 categorías en que fueron clasificadas las políticas de desarrollo económico local y la frecuencia con que los Planes de Desarrollo Comunal contienen alguna política de cada categoría.

En primer lugar, se observa que las políticas más frecuentes en las municipalidades son tres: las dirigidas a la capacitación, al emprendimiento y a la coordinación público-privada. Por otro lado, menos frecuentes, pero no necesariamente menos importantes (por ejemplo, en términos de inversión de recursos), son las políticas referidas a consumo, empleos municipales, compras públicas, personas de edad y relaciones o convenios internacionales. Al mismo tiempo, y a modo general, existen cuatro grandes dimensiones de las políticas, que se refieren a: i) empleo y capital humano; ii) institucionalidad; iii) desarrollo del sistema productivo comunal, y iv) emprendimiento y apoyo a las empresas. Para ordenar de mejor manera la exposición, a continuación se revisan brevemente las 40 categorías, enmarcadas en estas cuatro grandes líneas de acción. 


\section{Gráfico 3}

Chile: políticas de desarrollo económico local en los Planes de Desarrollo Comunal de las municipalidades, 2016

(En porcentajes de las municipalidades analizadas)

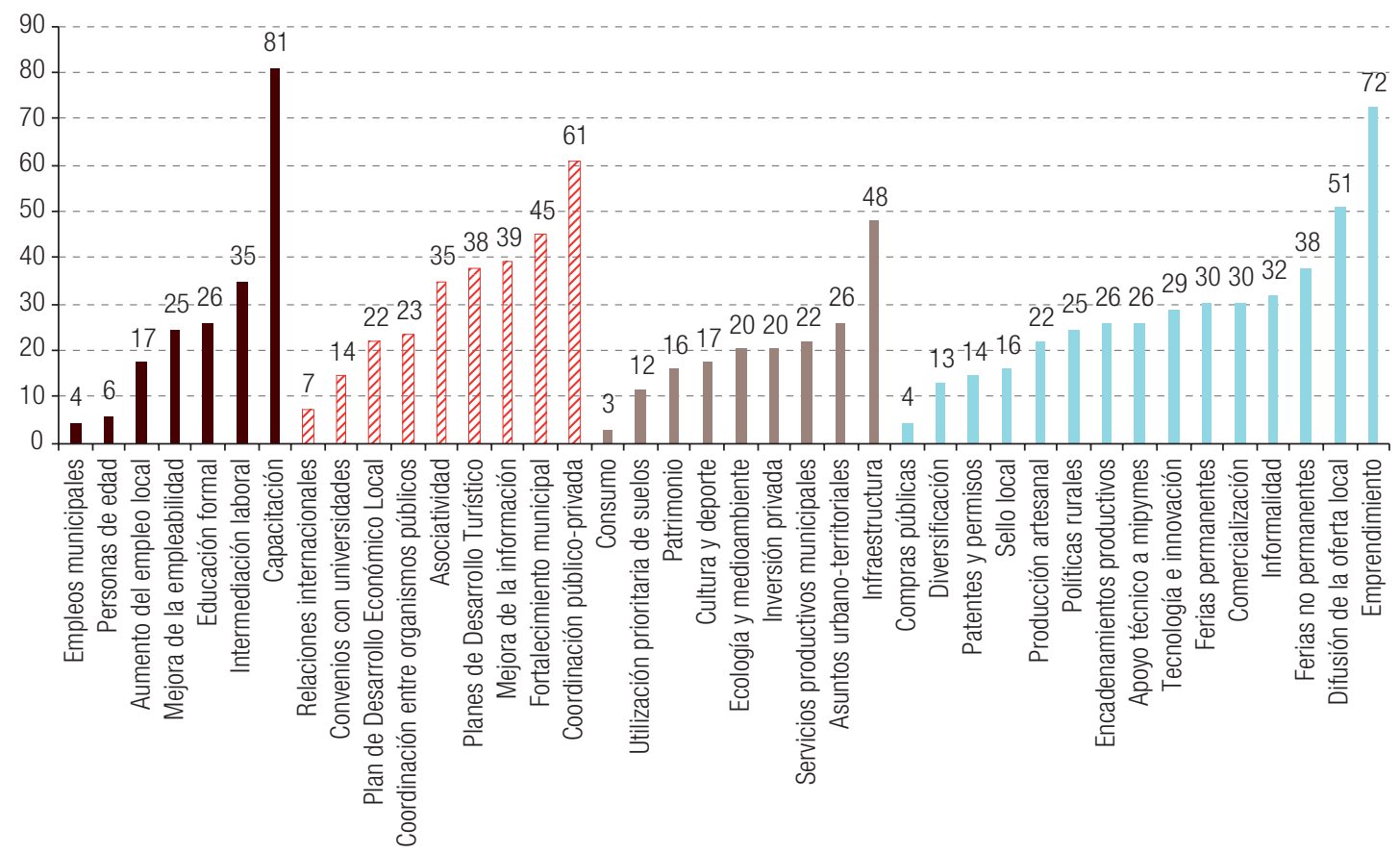

Empleo y capital humano

$\mathbb{Z}$ Institucionalidad

Sistema productivo comunal

Emprendimiento y apoyo a las empresas

Fuente: Elaboración propia, sobre la base de Planes de Desarrollo Comunal.

\section{Empleo y capital humano}

Las políticas asociadas a la capacitación son las que se proponen con mayor frecuencia, y se pueden referir a enseñar oficios para mejorar la empleabilidad de la fuerza de trabajo, favorecer el emprendimiento (por medio de la enseñanza de mecanismos para formalizar empresas, postular a fondos o gestionar los negocios), capacitar a los funcionarios municipales o capacitar a los líderes sociales en temas de desarrollo productivo. Como se muestra en el gráfico 3, el $81 \%$ de las municipalidades propone realizar actividades de capacitación. En este ámbito destacan especialmente las inversiones en construcción de espacios físicos municipales destinados exclusivamente a estos fines $^{7}$; la planificación de la oferta de capacitación de acuerdo con las necesidades de la estructura productiva local, para lo cual numerosas municipalidades hacen estudios del sistema productivo de la comuna, y la creación y puesta en marcha de los Organismos Técnicos de Capacitación (OTEC) ${ }^{8}$. Con respecto a este último punto, según datos del Servicio Nacional de Capacitación y Empleo (SENCE), en 2016 había 43 OTEC municipales, de los cuales 15 se encontraban en la Región Metropolitana (véase el gráfico 4). La importancia que tiene para las municipalidades el contar con estos organismos certificados se origina en la posibilidad de acceder a recursos públicos y privados para desarrollar las actividades de capacitación.

\footnotetext{
7 Entre las acciones que más se repiten está la creación de escuelas comunales de oficios o de capacitación.

8 LOS OTEC son instituciones acreditadas por el Servicio Nacional de Capacitación y Empleo (SENCE) con exclusividad para ejecutar actividades de capacitación que puedan imputarse a la Franquicia Tributaria y para ser contratadas por el Servicio a fin de llevar a cabo cursos financiados con recursos públicos. Para la acreditación de un OTEC municipal se debe cumplir con la norma chilena 2.728 y la norma ISO 9001:2008.
} 


\section{Gráfico 4}

Chile: Organismos Técnicos de Capacitación municipales por región administrativa, 2016

(En número de unidades)

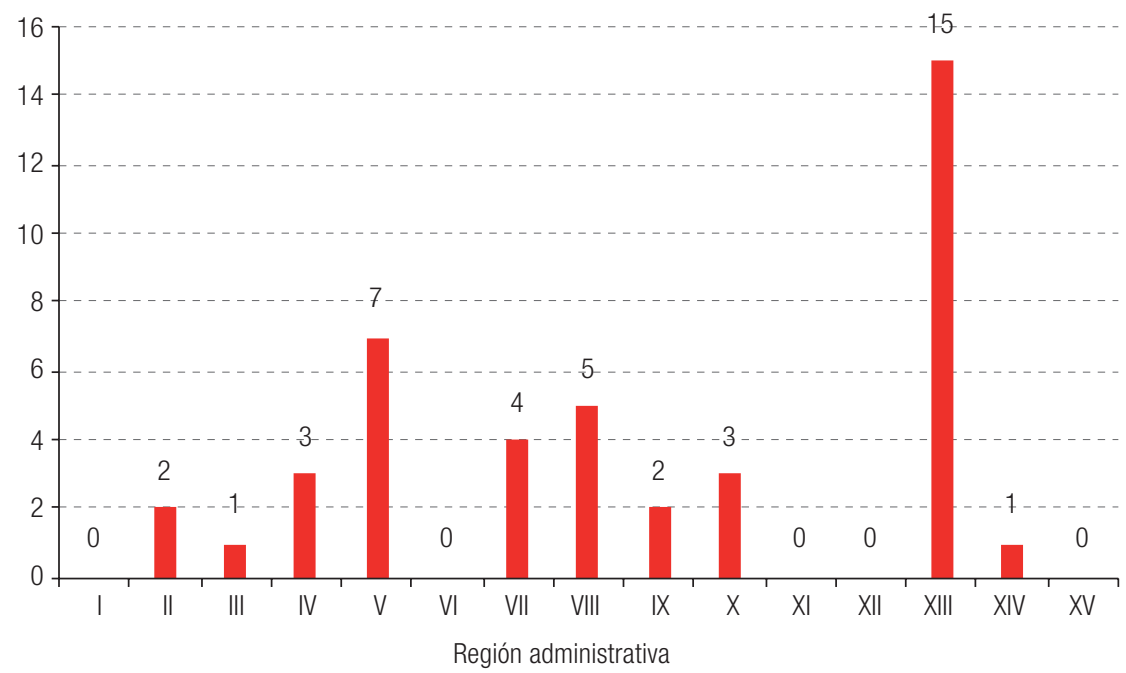

Fuente: Elaboración propia, sobre la base de datos del Servicio Nacional de Capacitación y Empleo (SENCE).

Otra política relacionada con el fortalecimiento del capital humano local es la referida a la educación formal, especialmente la formación técnica. Actualmente las municipalidades están a cargo de la administración de los liceos técnicos, lo que determina en parte que el 26\% de ellas proponga medidas sobre ese tema. Esto permite al mismo tiempo una planificación de la oferta de educación técnica de acuerdo con los requerimientos de la base productiva actual y futura y con las visiones acerca del sistema productivo comunal. En esta categoría se incluyen además las prácticas profesionales en convenio con el sector privado, los planes formativos en el ámbito del trabajo comunitario (como es el caso de la comuna de El Bosque), las medidas activas de nivelación de estudios, la educación preescolar y la creación de Centros de Formación Técnica municipales (en la comuna de Cerro Navia), entre otros aspectos.

El 25\% de las municipalidades cuentan con políticas para fortalecer la empleabilidad de la fuerza de trabajo, que tienen que ver con la capacitación y la educación formal o con otro tipo de actividades, como talleres de apresto laboral y certificaciones en oficios, competencias y calidad del recurso laboral (comunas de Villa Alemana y Cerro Navia).

La preocupación por el empleo se expresa también en las medidas que están dirigidas a disminuir la desocupación. Dentro de esta línea de política, las de mayor frecuencia son las tareas tradicionales de intermediación laboral, área que el 35\% de las municipalidades propone fortalecer. Aquí el foco de atención está en mejorar la labor de la Oficina Municipal de Intermediación Laboral (OMIL) a través de diversos mecanismos, como el programa de Fortalecimiento OMIL (FOMIL) del SENCE; la utilización de mejores tecnologías de información en la municipalidad y de la Bolsa Nacional de Empleo (BNE) para administrar la información relacionada con la intermediación laboral, y una activa vinculación de la municipalidad con las grandes empresas privadas para captar más y mejores oportunidades de empleo.

La disminución del desempleo es una de las prioridades locales de muchas municipalidades, especialmente en las llamadas comunas "dormitorio". El 17\% de las municipalidades han planteado varias líneas de acción al respecto, que incluyen incentivos para las empresas privadas que contraten mano de obra local (por ejemplo, el PLADECO de Maipú) o bonificaciones especiales en la evaluación de las propuestas formuladas por las firmas que postulen a licitaciones de la municipalidad y que 
incorporen personal de la comuna para desarrollar las labores programadas (comuna de Ovalle). También se han planteado medidas similares para paliar el desempleo que serían desarrolladas directamente por las municipalidades. El $4 \%$ de los planes analizados propone acciones en este sentido, entre las que se cuentan la contratación preferencial de mano de obra local para servicios provistos por la municipalidad (Maipú), el uso de la inversión pública en la comuna como un mecanismo de absorción de empleo (Temuco) y una idea interesante asociada al concepto de trabajo garantizado (Garzón y Guamán, 2015), esto es, que la municipalidad proporcione empleos de emergencia para las personas que los requieran en un programa continuo de mejoramiento urbano de la comuna (Melipilla). Un aspecto adicional es la focalización de algunas municipalidades en el tema del empleo con programas especialmente dirigidos a las mujeres y a los jóvenes, que cuentan con líneas específicas de financiamiento emanadas del gobierno central. A su vez, el $6 \%$ de las municipalidades consideran políticas centradas en la educación, la capacitación, el emprendimiento y la empleabilidad de las personas de edad.

\section{Institucionalidad}

Los temas que pertenecen al ámbito institucional son los siguientes: la elaboración de estudios y la creación de sistemas de información; el establecimiento de oficinas especializadas en desarrollo económico local; la incorporación de técnicos en la municipalidad; la planificación local relativa a la materia; la coordinación público-privada y entre instituciones públicas, y la promoción de asociaciones en el sector privado.

En las propuestas de capacitación y las de educación formal, así como en las políticas sobre empleabilidad, se repite la necesidad de contar con más información sobre la realidad productiva comunal. En este sentido, el 39\% de las municipalidades proponen medidas concretas para mejorar la calidad de la información con la que cuentan, que incluyen tanto la ejecución de estudios sobre la realidad productiva como la creación de sistemas de información permanentes. En este segundo caso, son frecuentes las propuestas para realizar catastros de empresas y emprendimientos -incluidas las actividades formales e informales y los distintos tamaños de empresa-, así como de fuentes de empleo. En los nuevos escenarios, contar con información actualizada y completa se convierte en un elemento central para la evaluación permanente de los desafíos.

El 45\% de las municipalidades cree necesario avanzar en el fortalecimiento del área de desarrollo económico local. En este sentido, y a pesar de que no está explícitamente incluida en la Ley Orgánica Constitucional de Municipalidades como una de las unidades de la organización municipal, muchas municipalidades han empezado a crear la Dirección de Desarrollo Económico Local (DIDEL) con el fin de potenciar las acciones a ese respecto y reunir en una misma unidad a oficinas que antes estaban dispersas en distintas direcciones. Mediante una revisión de los sitios web de 158 municipalidades de Chile, de comunas con más de 20.000 habitantes, se observó que el 10\% de las municipalidades cuenta con una DIDEL (véase el gráfico 5), creada ya sea de modo formal por medio de un reglamento o de modo fáctico. Sin embargo, la mayoría de las municipalidades $(74,7 \%)$ aún tiene radicadas las labores de desarrollo económico local en las Direcciones de Desarrollo Comunitario (DIDECO), ya sea como un departamento o como oficinas temáticas destinadas no solo al desarrollo económico local ${ }^{9}$.

\footnotetext{
9 En este ordenamiento se dio prioridad a la investigación sobre la ubicación del fomento productivo como actividad principal o nuclear del desarrollo económico local.
} 


\section{Gráfico 5}

Chile: direcciones donde se alojan las tareas de fomento productivo en las municipalidades, 2016

(En porcentajes)

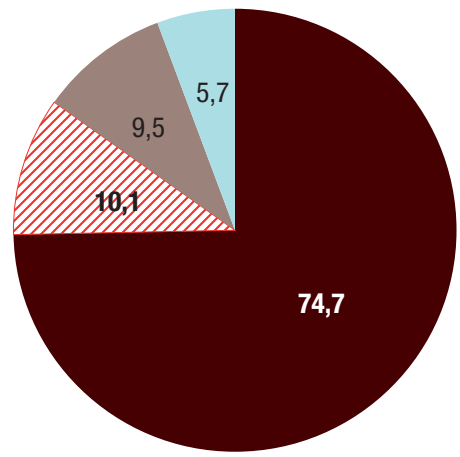

Dirección de Desarrollo
$\begin{array}{lll}\text { Comunitario } & \text { Dirección de Desarrollo } & \text { Secretaría Comunal } \\ \text { Económico Local } & \text { de Planificación }\end{array}$

Fuente: Elaboración propia, sobre la base de los sitios web de las municipalidades.

En algunos PLADECO el fortalecimiento municipal también incluye la incorporación de profesionales especializados en la asesoría técnica a emprendedores y empresas que la necesiten, y que tengan un conocimiento cabal de los planes y programas de gobierno a los cuales las empresas y las municipalidades pueden postular para obtener recursos.

La categoría de fortalecimiento municipal del desarrollo económico-productivo está íntimamente ligada a la creación de un Plan de Desarrollo Económico Local (PLADEL) ${ }^{10}$, tarea a la que han declarado estar abocadas un $22 \%$ de las municipalidades consultadas. Otro tipo de política de planificación corresponde a la elaboración del Plan de Desarrollo Turístico (PLADETUR), actividad que es considerada por el $38 \%$ de las municipalidades. Es interesante observar, si se contabilizan las municipalidades que elaboran un PLADEL y las que elaboran un PLADETUR, que solo el 9\% propone el desarrollo de ambos planes, mientras que el 29\% formula propuestas relativas al turismo, sin insertarlas en una estrategia más general de desarrollo económico local.

Para la elaboración de estos planes - tarea que debería conducir eventualmente la DIDEL - es necesario tener en cuenta no solo los puntos de vista y el campo de acción de la municipalidad, sino también los de los demás organismos públicos, la sociedad civil, las entidades gremiales e incluso las demás unidades municipales. Por esto, el $61 \%$ de las municipalidades propone explícitamente formular políticas orientadas a fortalecer la coordinación público-privada. Además, el 23\% plantea propuestas orientadas a mejorar la coordinación con otros organismos del Estado e incluso con otras unidades municipales cuyos ámbitos de acción se intersecan con las actividades de desarrollo económico y productivo, lo que ha sido señalado previamente como un elemento esencial para la eficacia de las políticas locales de fomento productivo en Chile (Bravo, 2012). La mayoría de las municipalidades que proponen la coordinación público-privada se refieren a mesas de trabajo temáticas para diferentes rubros.

\footnotetext{
${ }^{10}$ LOS PLADEL son documentos que 15 de las municipalidades analizadas proponen desarrollar de forma complementaria a Ios PLADECO, y que, desde el punto de vista del presente estudio, reflejan una preocupación específica por elaborar una propuesta más acabada respecto del desarrollo económico local. No deben confundirse con las secciones de desarrollo económico o productivo de los PLADECO, que son las principales fuentes de información del presente análisis. La diferencia entre estos dos planes radica en que la sección de desarrollo económico local del PLADECO plantea una visión de mediano o largo plazo, mientras que en el PLADEL se operacionaliza lo planteado en el PLADECO, ya que se estudia la viabilidad de las acciones concretas.
} 
Al mismo tiempo, varios PLADECO sugieren la conformación de comités en torno a los temas de la Base Productiva Comunal en el seno de los Consejos Comunales de Organizaciones de la Sociedad Civil ${ }^{11}$. El desafío está en instalar espacios permanentes de coordinación que puedan otorgar una perspectiva a largo plazo y que contengan la visión tanto de la municipalidad como de las asociaciones gremiales y la sociedad civil.

Uno de los problemas detectados en relación con la coordinación público-privada es que en ocasiones las entidades productivas no cuentan con organismos gremiales que las reúnan. Para solucionar este problema, la municipalidad puede potenciar la asociatividad en sus diversas formas, lo que se propone en el 35\% de los Planes de Desarrollo Comunal. Esto implica el fomento de formas asociativas como las cooperativas, las cámaras, las asociaciones gremiales o las simples instancias coordinadoras. Por ejemplo, uno de los problemas que se señaló en las instancias de participación en Quinta Normal se refiere a que el concepto de crear asociaciones carece de oportunidades de legitimación, lo que significa que es responsabilidad de la municipalidad generarlas, incentivando al mismo tiempo la formación de asociaciones gremiales entre los agentes productivos, es decir, debería actuar como un "legitimador".

El promover la creación de asociaciones, en particular las de trabajadores y microempresarios a través de la conformación de empresas cooperativas, es también una alternativa que han empezado a proponer algunas municipalidades (como Recoleta y Maipú), a menudo con la activa colaboración de la División de Asociatividad y Economía Social (DAES) del Ministerio de Economía, Fomento y Turismo.

Un tipo especial de coordinación público-privada se establece entre las municipalidades y los entes internacionales, y entre ellas y las universidades. Mientras un $7 \%$ de las municipalidades propone políticas relacionadas con el primer caso, la cifra llega al 14\% respecto del segundo. Los acuerdos internacionales se llevan a cabo con gobiernos locales de otros países en torno a coordinaciones temáticas en las áreas de turismo o ciudades-puerto, entre otras. Los convenios con las universidades se plantean principalmente para atraer estudiantes en práctica y para realizar estudios específicos sobre la realidad productiva local.

\section{Desarrollo del sistema productivo comunal}

En este ámbito, las propuestas fundamentales se relacionan con la promoción de inversiones privadas, la creación de infraestructura productiva, las políticas urbano-territoriales y de uso del suelo, los servicios productivos municipales y las políticas relativas al medioambiente -elaboradas desde un punto de vista económico-productivo-, el patrimonio, la cultura y el consumo.

Un tema relevante es la inversión privada, pues un 20\% de las municipalidades plantean políticas relacionadas con él. La mayoría de las que lo hacen resaltan la necesidad de diseñar mecanismos para atraer inversiones a la comuna, lo que a su vez se relaciona tanto con la difusión de oportunidades de inversión como con los incentivos contenidos en el Plan Regulador Comunal. Respecto de esto último, la utilización prioritaria del suelo para ciertos tipos de inversión es relevada por el 12\% de las municipalidades e involucra la definición de parques tecnológicos, parques industriales y zonas industriales, agroindustriales y comerciales - en algunos casos con infraestructura proporcionada por la municipalidad - o zonas donde el valor de la patente es menor, lo que supone que este parámetro se utiliza como un mecanismo de ordenamiento de la actividad comercial y productiva de la comuna.

\footnotetext{
${ }^{11}$ El Consejo Comunal de Organizaciones de la Sociedad Civil (COSOC) es un organismo comunal establecido en la Ley Orgánica Constitucional de Municipalidades (art. 94) que funciona como contrapeso del alcalde y la municipalidad para la elaboración y aprobación de los instrumentos de la gestión municipal (PLADECO, Plan Regulador Comunal, presupuesto), entre otros asuntos de interés comunal.
} 
Por otra parte, la necesidad de que las municipalidades proporcionen infraestructura para el desarrollo es rescatada por el 33\% de ellas. Estas políticas se relacionan principalmente con la infraestructura turística (como señalética, miradores, borde costero, parques y museos), pero también consideran la provisión de infraestructura productiva a los sectores rurales (en la forma de diques, embalses, riego y energía, entre otras) y a las actividades comerciales (ferias, mercados y otras). Relacionada con la infraestructura está la provisión de servicios productivos municipales (22\%), que corresponden a los servicios directamente suministrados por la municipalidad o a las actividades productivas realizadas por ellas. En esta categoría se incluyen muy diversas propuestas, como la conformación de un Centro de Formación Técnica municipal (comuna de El Bosque), la construcción de una planta de compostaje (Los Ángeles), la realización de tours municipales por la ciudad (Temuco) y la habilitación de zonas de camping municipales (Melipilla), entre otras iniciativas.

Las políticas de desarrollo económico propuestas en los Planes de Desarrollo Comunal están en estrecha relación con las definiciones estratégicas sobre el uso del suelo de los Planes Reguladores Comunales e incluyen políticas de tipo urbano-territoriales, categoría en la que propone actividades el $26 \%$ de las municipalidades. A este tipo de políticas corresponde la revitalización de barrios, y sectores céntricos y comerciales. Otra política relacionada con lo anterior es la puesta en valor del patrimonio (relacionada con el catastro y la revitalización de sitios patrimoniales), aspecto rescatado por el $16 \%$ de los gobiernos locales en sus planes de desarrollo económico-productivo y que se vincula en la gran mayoría de los casos a la dimensión turística de la comuna.

En relación con el tema patrimonial, las políticas que atañen al arte y la cultura también han sido propuestas por el $16 \%$ de las municipalidades, y se vinculan en general al turismo y a las actividades que se llevan a cabo en la época estival o en fechas especiales.

Para concluir, hay dos últimos temas asociados al desarrollo del sistema productivo comunal: las políticas medioambientales y las políticas de consumo. Mientras el 20\% de las municipalidades consideran al aspecto medioambiental o de desarrollo sostenible dentro de sus políticas de desarrollo económico-productivo, solo un 3\% incluye políticas de consumo. Las primeras giran en torno a la creación de una industria del reciclaje, la revitalización de parques y áreas verdes, la inclusión de energías renovables no convencionales en la estructura productiva local (y en la propia municipalidad), el fortalecimiento de la fiscalización ambiental, la difusión de buenas prácticas sostenibles para los negocios y el impulso a la agricultura orgánica, entre otras. En cuanto al consumo, la preocupación radica sobre todo en la protección de los derechos del consumidor a través de la implementación de una Oficina del Consumidor en colaboración con el Servicio Nacional del Consumidor (SERNAC) ${ }^{12}$.

Cabe destacar que varias de las políticas mencionadas (especialmente las de patrimonio, cultura, medioambiente y urbanismo) son en la realidad más numerosas de lo que aquí se ha indicado. Esto se debe a que en varios de los PLADECO se elaboran propuestas específicas sobre los temas urbano, medioambiental, cultural o de otro tipo en secciones ad hoc, separadas de las que tratan sobre las políticas de desarrollo económico-productivo que se analizan en este artículo. Aun así, no deja de ser interesante la capacidad de algunas administraciones de vincular varias de estas medidas, lo que demuestra un entendimiento más completo del proceso de desarrollo económico local.

\section{Emprendimiento y apoyo a las empresas}

La categoría de emprendimiento se encuentra presente en el $72 \%$ de las municipalidades y conforma, después de la de capacitación, el área de intervención de la política de desarrollo económico local más extendida. Las políticas de fomento del emprendimiento comprenden las incubadoras de

\footnotetext{
${ }^{12}$ Este tipo de convenios de colaboración se encuentra vigente en varias municipalidades del país y supone acercar la normativa sobre derechos del consumidor a las comunas con menor acceso a las instituciones del gobierno central.
} 
negocios, el asesoramiento para la postulación a fondos de fomento productivo públicos y privados, la provisión de información sobre programas de gobierno y trámites legales, y los talleres de formación y asistencia técnica, entre otras. El apoyo técnico directo de las municipalidades a las pymes, que es propuesto por el $26 \%$ de ellas, implica contar con profesionales que puedan acompañar y asesorar los emprendimientos y ha sido mencionado como un elemento clave tanto en la categoría de emprendimiento como en la de apoyo técnico.

El 13\% de las municipalidades incluye políticas de diversificación productiva, lo que supone un apoyo al emprendimiento focalizado en actividades innovadoras en el territorio y la identificación y difusión de posibles nuevos nichos productivos para emprendedores de la zona.

Otro tipo de políticas dentro de este ámbito es el que se refiere a tecnología e innovación, que es uno de los focos de acción del 29\% de las municipalidades. Esta categoría incluye, por ejemplo, políticas de alfabetización digital -incluidas capacitaciones-, creación de parques tecnológicos y elaboración de programas de transferencia tecnológica en convenio con universidades.

El $4 \%$ de las municipalidades plantea fortalecer los mecanismos de compra pública y el $26 \%$ propone políticas asociadas a encadenamientos productivos. Mientras en el primer caso las actividades se centran en capacitaciones para convertirse en proveedor del sistema público a través del portal nacional de ChileCompra, en el caso de las políticas orientadas a fortalecer los encadenamientos productivos se considera la ejecución de estudios para evaluar la realidad productiva local, y el fomento de las mipymes locales como proveedoras de las grandes empresas ubicadas en la comuna.

Otra forma en que las municipalidades han abordado el impulso a la actividad productiva local ha sido a través de políticas de difusión de la oferta local, categoría a la que adscribe el $51 \%$ de las municipalidades. Este tipo de política considera mayoritariamente la difusión de la oferta turística a través de Internet, folletos y planes de difusión, y el desarrollo de plataformas web en que se informe sobre el comercio y los productos a escala del barrio y la comuna. Además de la difusión, el 30\% de las municipalidades propone actividades destinadas a mejorar la comercialización de los productos locales, lo que implica en la mayoría de los casos la habilitación de sitios permanentes para la muestra y venta de estos productos, especialmente orientados a artesanos, emprendedores, microempresarios y productores agrícolas.

Existen políticas similares a las que promueven la comercialización de productos locales, que tienen que ver con la producción artesanal y el sector rural, las cuales son abordadas por el 22\% y el 25\% de las municipalidades, respectivamente. En el caso de la producción artesanal, las políticas van dirigidas en su mayoría a fortalecer la comercialización mediante puntos de venta permanentes. Respecto de las políticas rurales, es destacable la voluntad de ampliar la cobertura del Programa de Desarrollo de Acción Local (PRODESAL) ${ }^{13}$, dado que las demandas son muy variadas y apuntan a las necesidades concretas de cada comuna particular.

Otra categoría de política tiene que ver con la implementación de sellos locales para la producción y los servicios, propuesta por el $16 \%$ de las municipalidades. Esto incluye planes cuyo objeto es aumentar la certificación de denominación de origen ${ }^{14}$ y las marcas de certificación en diversos rubros, como la producción silvoagropecuaria y el turismo.

\footnotetext{
${ }^{13}$ El Programa de Desarrollo de Acción Local (PRODESAL) del Instituto de Desarrollo Agropecuario (INDAP) - dependiente del Ministerio de Agricultura - está orientado a mejorar la producción agrícola y ganadera de los pequeños productores a través de la asesoría técnica y el acceso a fondos de inversión. El PRODESAL es un programa ejecutado por municipalidades o, excepcionalmente, entidades privadas a las que el INDAP transfiere recursos mediante asesorías técnicas o inversión -en el marco de un convenio o contrato-, que se complementan con los recursos que aportan dichas entidades ejecutoras.

${ }^{14}$ Las denominaciones de origen protegen los productos originarios de un país, una región o una localidad, siempre que su calidad, reputación u otra característica se pueda atribuir al origen geográfico. Además, debe haber factores naturales y humanos que incidan en la caracterización del producto. En la actualidad, la protección que implica el reconocimiento de una denominación de origen puede alcanzar cualquier tipo de producto, no solo agrícola, siempre que cumpla con los requisitos previstos en la Ley de Propiedad Industrial, en su reglamento o en otras normas especiales.
} 
Relacionados con la exhibición y comercialización de los productos locales están los mercados no permanentes (ferias periódicas) y los mercados permanentes (ferias libres, ferias persas, ferias de abastos y mercados municipales, entre otros), que corresponden a categorías de política sobre las cuales existen propuestas por parte del $28 \%$ y el $31 \%$ de las municipalidades, respectivamente. En cuanto a las ferias periódicas, el objetivo es mostrar y ofrecer los productos de origen local (elaborados, por ejemplo, por emprendedores o artesanos), a veces en coordinación con ferias costumbristas u otras actividades organizadas por la municipalidad. Este tipo de política se orienta principalmente a productos que no son de consumo habitual. En la categoría de mercados permanentes las propuestas van encaminadas a instalar mercados municipales en las comunas en que no existen; ordenar, regular y revitalizar las ferias libres, junto con instalar infraestructura sanitaria y de limpieza, y redireccionar el comercio ambulante y estacionario (callejero) hacia ferias libres y ferias persas, además de fiscalizar, especialmente en los lugares donde se venden alimentos.

Las dos últimas categorías se refieren a las actividades económicas informales (32\%) y a la política de patentes y permisos municipales (14\%), que se relacionan entre sí y con las políticas de mercados y ferias permanentes. En cuanto a la informalidad, un tercio de las municipalidades que incorporan esta categoría en su planificación otorgan una alta relevancia a la formalización de los emprendimientos y, especialmente, del comercio ambulante. Una de las formas que adquiere esta iniciativa es la ayuda que se proporciona para la formalización de actividades mediante la constitución de microempresas familiares ${ }^{15}$. En general se considera hacer catastros de los comerciantes de la vía pública, y se incentiva su formalización, junto con ofrecerles capacitación y la posibilidad de reubicarse y, en algunos casos, la reconversión productiva. También se incluye la fiscalización, ya que los procedimientos de formalización se efectúan mediante ordenanzas municipales. Unidas a lo anterior se encuentran algunas políticas insertas en la categoría de patentes y permisos municipales, que tienen relación con el financiamiento de los trámites municipales necesarios para que los microempresarios comerciales formalicen sus actividades. Finalmente, en algunas municipalidades existe la voluntad de mejorar los procedimientos para otorgar patentes y permisos mediante la elaboración y simplificación de los protocolos respectivos, la habilitación de una ventanilla única para el inicio de actividades comerciales, la revisión y eventual modificación de las ordenanzas comerciales de la comuna y la difusión de los beneficios potenciales de la formalización.

\section{Características comunales}

En esta sección se avanza hacia la formulación de una hipótesis analítica destinada a verificar eventuales conexiones entre las características de las municipalidades y las actividades que cada gobierno local se propone hacer (expresadas en el número de categorías de actividades incluidas en los PLADECO).

Para eso se consideró una muestra de 20 comunas, que se extrajo de las 69 estudiadas. Después de ordenar las 69 municipalidades según el número de categorías de los respectivos PLADECO, se conformó la muestra eligiendo a las 10 municipalidades o comunas cuyos planes tenían el mayor número de categorías y a las 10 en que estos presentaban el número más bajo. Esta modalidad de elaboración de la muestra tiene por objeto extremar las diferencias entre las municipalidades con el fin de poner de relieve más fácilmente las características que tienen mayor relevancia en la elaboración de los PLADECO más complejos.

\footnotetext{
${ }^{15}$ La ventaja de crear una microempresa familiar se refiere a la posibilidad de optar a créditos o financiamiento públicos, y a la ausencia de limitaciones de funcionamiento en relación con la zonificación comercial o industrial incluida en las respectivas ordenanzas municipales y con las autorizaciones que previamente deben otorgar las autoridades sanitarias u otras que exija la ley, además de poder acceder a un proceso simplificado de inicio de actividades.
} 
Dentro de las 10 municipalidades más diversificadas, se encuentran, en orden decreciente, las siguientes: La Florida (21), Los Andes (21), Iquique (20), Temuco (20), Coquimbo (19), Santiago (19), Villa Alemana (19), Aysén (18), Lebu (18) y Maipú (18). Por otro lado, las 10 comunas que abarcan menos categorías en su planificación son, en orden decreciente: Arica (5), Estación Central (4), Quilpué (4), Rancagua (4), Renca (4), Castro (3), Quillota (3), San Felipe (3), Ñuñoa (2) y Puente Alto (2).

El método utilizado consiste en una regresión multivariable donde la variable dependiente es la cantidad de categorías y las variables independientes, clasificadas en tres grupos, son:

- De la comuna: si es capital regional, capital provincial o pertenece a la Región Metropolitana, población total ${ }^{16}$, porcentaje de pobreza de ingresos y porcentaje de pobreza multidimensional ${ }^{17}$.

- De la municipalidad: si la orientación política del alcalde que firma el PLADECO es de derecha o de izquierda ${ }^{18}$ y el presupuesto municipal total ${ }^{19}$.

- Del PLADECO: el año de elaboración, su duración, si fue hecho o no por una consultora externa y si hubo participación ciudadana en ese proceso ${ }^{20}$.

Dado el reducido número de sujetos que conforman la muestra, los resultados que se sintetizan en el cuadro 1 no deben ser considerados como pruebas definitivas de las relaciones entre las características de las municipalidades y las acciones que estas planifican, sino como sugerencias preliminares para el estudio de dichas relaciones.

\section{Cuadro 1}

Chile: características relacionadas con la cantidad de categorías relativas a las políticas de desarrollo económico local (Coeficientes y desuiaciones estándar)

\begin{tabular}{|c|c|c|c|c|}
\hline & (1) & (2) & (3) & (4) \\
\hline \multicolumn{5}{|l|}{ Comuna } \\
\hline Capital regional & $\begin{array}{c}-19,68 \\
(13,03)\end{array}$ & $\begin{array}{r}-17,75^{*} \\
(8,87)\end{array}$ & $\begin{array}{r}-17,81^{*} \\
(8,25)\end{array}$ & $\begin{array}{r}-17,06^{*} \\
(8,14)\end{array}$ \\
\hline Capital provincial & $\begin{array}{c}2,25 \\
(10,46)\end{array}$ & - & - & - \\
\hline Pertenencia a la Región Metropolitana & $\begin{array}{r}-12,67 \\
(9,55)\end{array}$ & $\begin{array}{r}-13,14 \\
(8,71)\end{array}$ & $\begin{array}{c}-13,17 \\
(8,20)\end{array}$ & $\begin{array}{c}-12,78 \\
(8,12) \\
\end{array}$ \\
\hline Población total & $\begin{array}{c}-0,04 \\
(0,03)\end{array}$ & $\begin{array}{c}-0,04 \\
(0,03)\end{array}$ & $\begin{array}{c}-0,04 \\
(0,02)\end{array}$ & $\begin{array}{c}-0,04 \\
(0,02)\end{array}$ \\
\hline Pobreza de ingresos & $\begin{array}{c}0,01 \\
(0,63)\end{array}$ & $\begin{array}{l}-0,04 \\
(0,03)\end{array}$ & - & - \\
\hline Pobreza multidimensional & $\begin{array}{c}0,51 \\
(0,66)\end{array}$ & $\begin{array}{c}0,57 \\
(0,56)\end{array}$ & $\begin{array}{c}0,55 \\
(0,38)\end{array}$ & $\begin{array}{c}0,54 \\
(0,38)\end{array}$ \\
\hline \multicolumn{5}{|l|}{ Municipalidad } \\
\hline Orientación política & $\begin{array}{c}5,99 \\
(8,18)\end{array}$ & $\begin{array}{c}4,80 \\
(5,69)\end{array}$ & $\begin{array}{c}4,92 \\
(4,68)\end{array}$ & $\begin{array}{c}5,37 \\
(4,61)\end{array}$ \\
\hline Presupuesto total & $\begin{array}{l}0,48^{\star *} \\
(0,19)\end{array}$ & $\begin{array}{c}0,47^{\star *} \\
(0,16)\end{array}$ & $\begin{array}{c}0,47^{\star \star} \\
(0,15)\end{array}$ & $\begin{array}{c}0,46^{\star *} \\
(0,15)\end{array}$ \\
\hline
\end{tabular}

\footnotetext{
${ }^{16}$ De 2016, según el Instituto Nacional de Estadísticas.

17 Ambas según los datos de la Encuesta de Caracterización Socioeconómica Nacional (CASEN) de 2015.

${ }^{18}$ La derecha política incluye a la Alianza por Chile, y la izquierda, a la Nueva Mayoría. En el caso de los alcaldes independientes, se consideró el pacto por el que fueron candidatos o las militancias previas en el caso de presentarse fuera de un pacto. La información proviene del Servicio Electoral de Chile (SERVEL) y de medios de prensa.

${ }^{19}$ De 2016, según el Sistema Nacional de Información Municipal (SINIM).

${ }^{20}$ Esto último se determinó según la información proporcionada por la Subsecretaría de Desarrollo Regional y Administrativo (SUBDERE).
} 
Cuadro 1 (conclusión)

\begin{tabular}{|c|c|c|c|c|}
\hline & (1) & (2) & (3) & (4) \\
\hline \multicolumn{5}{|l|}{ PLADECO } \\
\hline Año & $\begin{array}{l}-1,11 \\
(0,94)\end{array}$ & $\begin{array}{l}-1,10 \\
(0,88)\end{array}$ & $\begin{array}{l}-1,09 \\
(0,83)\end{array}$ & $\begin{array}{l}-1,23 \\
(0,81)\end{array}$ \\
\hline Duración & $\begin{array}{l}-1,98 \\
(2,69)\end{array}$ & $\begin{array}{l}-1,54 \\
(1,65)\end{array}$ & $\begin{array}{l}-1,54 \\
(1,56)\end{array}$ & $\begin{array}{l}-1,47 \\
(1,54)\end{array}$ \\
\hline Consultora & $\begin{array}{c}11,24 \\
(6,57)\end{array}$ & $\begin{array}{c}10,72^{*} \\
(5,74)\end{array}$ & $\begin{array}{c}10,67^{*} \\
(5,28)\end{array}$ & $\begin{array}{c}9,95^{*} \\
(5,18)\end{array}$ \\
\hline Participación ciudadana & $\begin{array}{l}-7,55 \\
(9,07) \\
\end{array}$ & $\begin{array}{l}-7,23 \\
(8,40)\end{array}$ & $\begin{array}{c}-7,25 \\
(7,90) \\
\end{array}$ & - \\
\hline Constante & $\begin{array}{c}2227,06 \\
(1898,41)\end{array}$ & $\begin{array}{c}2205,75 \\
(1779,26)\end{array}$ & $\begin{array}{c}2197,71 \\
(1667,82)\end{array}$ & $\begin{array}{c}2476,07 \\
(1627,06)\end{array}$ \\
\hline $\mathrm{R}^{2}$ ajustado & 0,05 & 0,16 & 0,25 & 0,27 \\
\hline
\end{tabular}

Fuente: Elaboración propia.

Nota: $\quad{ }^{*}$ significativo al $10 \%,{ }^{* *}$ significativo al $5 \%,{ }^{\star \star *}$ significativo al $1 \%$.

Las distintas columnas del cuadro representan cuatro conjuntos diferentes de variables dependientes. Aunque varíe la lista de dichas variables, el resultado no cambia: en todos los casos las únicas variables significativas son el presupuesto municipal, la consultoría externa y el hecho de ser capital regional.

La correlación positiva del número de categorías con el presupuesto es esperable y puede ser interpretada como que las municipalidades con mayores recursos son más proclives a planificar actividades que abarcan un abanico más amplio de categorías de acción.

También existe una correlación positiva con las empresas consultoras. En este caso, sin embargo, la lectura del fenómeno puede resultar menos fácil de hacer. Una interpretación positiva sugeriría que la presencia de una consultora como un refuerzo de la capacidad de planificación de las municipalidades permitiría a las alcaldías que cuentan con este recurso ampliar el espectro de acciones consideradas en sus planes. Una lectura menos positiva podría apuntar a que el incremento en el número de categorías de los PLADECO es un reflejo de que las consultoras, al no tener responsabilidades directas en la implementación de los planes que diseñan, proponen todas las acciones que les parecen teóricamente interesantes sin el filtro más estricto de lo que la municipalidad puede efectivamente hacer.

La tercera variable que tiene correlación con el número de categorías - en este caso de signo negativo - es la condición de capital regional. Una posible explicación podría buscarse en el efecto de "desplazamiento" de parte de las instituciones nacionales de fomento. Dichas instituciones concentran en las capitales regionales una parte preponderante de las acciones de apoyo al sector productivo que realizan fuera de Santiago, y esto, de alguna manera, puede inducir a los gobiernos locales a desinteresarse del problema del desarrollo económico local para concentrarse en otras prioridades.

Por último, resulta interesante intentar una interpretación de los resultados respecto de las variables no significativas. En primer lugar, la intensidad del esfuerzo planificado no parece tener color político definido, pues la pertenencia de los alcaldes a las distintas alianzas no es una variable que explique la mayor complejidad de los PLADECO en cuanto al desarrollo económico local. Tampoco es relevante el nivel de pobreza, lo cual sugeriría que la motivación para programar más categorías de acciones está relacionada con un conjunto amplio de necesidades.

El hecho de que la participación no explique la complejidad de los planes significa que estos pueden ser complejos tanto en municipalidades que emprenden estrategias participativas como en otras que no lo hacen, lo que indicaría que dichas estrategias no son un obstáculo para elaborar planes más complejos. 
Respecto a la duración, podría esperarse que cuanto más amplio es el horizonte de planificación, mayor es el número de categorías. El hecho de que esa variable no sea significativa da a entender que hay una cantidad importante de municipalidades que plantean planes de actividades muy diversificados para plazos reducidos, lo que supondría algún grado de ingenuidad o voluntarismo en las propuestas. Finalmente, el año de formulación del plan podría ser indicio de un proceso de aprendizaje, pues, mientras más antiguo sea el plan, existe una mayor probabilidad de que esté desactualizado y no haya incorporado sucesivos aprendizajes mediante la formulación de otros planes. Esto, sin embargo, no se verifica empíricamente.

\section{Conclusiones}

El análisis de la política local de desarrollo económico ha sido hasta ahora un campo poco explorado en Chile y dentro de la disciplina económica. Esto se debe probablemente al déficit de información y datos con los que se cuenta para la investigación sobre la estructura económica y productiva local, y a la concepción fundamentalmente nacional de la comprensión sobre los procesos de desarrollo económico. De esta manera, la presente investigación aspira a contribuir al entendimiento de las políticas de desarrollo económico local que se realizan en la práctica.

Del análisis de las políticas de desarrollo económico local incorporadas en los Planes de Desarrollo Comunal se pueden extraer varias reflexiones y conclusiones. En primer lugar, se debe notar que el total de políticas detectadas en el conjunto de municipalidades sobrepasa ampliamente lo que realiza cada una de forma individual. De las 40 categorías de política identificadas, las municipalidades consultadas rescatan en promedio 16, es decir, un $40 \%$ de las posibilidades de política existentes. Por otra parte, la presencia de estas 40 categorías da cuenta de la posibilidad de trascender la concepción asistencialista de la política de desarrollo económico local, que en la práctica se reduce a prestar ayuda focalizada a personas desempleadas mediante talleres, capacitaciones, intermediación laboral y apoyo para el microemprendimiento, que, como señalan algunos PLADECO, muchas veces no constituyen soluciones estables para la situación económica de las familias.

Un segundo elemento novedoso en la gestión de las políticas de desarrollo económico local se relaciona con la creación de direcciones o departamentos especializados en el tema, lo que supone trasladar esa responsabilidad desde las actuales Direcciones de Desarrollo Comunitario (DIDECO) a las recientemente creadas Direcciones de Desarrollo Económico Local (DIDEL) o unificar esa tarea en Departamentos de Desarrollo Económico Local dentro de alguna dirección.

Un tercer aspecto relevante concierne al horizonte de planificación, que en las municipalidades alcanza un promedio de 4,6 años. Para garantizar una alineación más clara con los planes de desarrollo productivo nacionales y regionales (que superan los diez años de plazo), sería útil explorar la posibilidad de definir un mínimo de diez años como horizonte de acción de los PLADECO, en combinación con planes municipales - o programas de gobierno - de cuatro años de duración que cubrieran el período de mandato, siempre orientados por la planificación estratégica definida en el Plan de Desarrollo Comunal. A la hora de analizar este nuevo tipo de enfoque, se advierte que también son relevantes las propuestas de planificación a largo plazo y una mayor injerencia del gobierno local en los aspectos institucionales del desarrollo, que, sin duda y como lo dejan en claro los Planes de Desarrollo Comunal, consideran la participación ciudadana y la coordinación activa con los agentes productivos locales como ejes de la planificación y las propuestas en torno al tema.

En cuarto lugar, si bien muchas municipalidades delegan la elaboración de los PLADECO en consultoras externas, hay otras que los desarrollan con recursos propios. La evaluación de la calidad con la que se llevan a cabo estos planes requeriría de un análisis que evidentemente sobrepasa las 
posibilidades del presente estudio, pero es importante reconocer que en muchas municipalidades existen las bases para desempeñar de forma directa esta labor de planificación, que debería apuntar a generar lazos de confianza entre los gobiernos locales y la ciudadanía y a obtener el respaldo y la legitimidad necesarios para llevar adelante las políticas. Si participan consultoras en la preparación de los planes, no tendrían que hacerlo tanto respecto del contenido, sino apoyando el proceso de elaboración.

Por último, cabe destacar que las características municipales también importan. Entre ellas, las que aparentemente tienen mayor relevancia al explicar las labores de planificación más articuladas son el tamaño del presupuesto municipal, la presencia de empresas consultoras y la condición de ser capital regional.

Considerando los hallazgos de la investigación es posible retomar la cuestión inicial que motiva el estudio y plantear una respuesta preliminar. Ante la pregunta sobre la medida en que las políticas de desarrollo económico local se circunscriben en Chile a una concepción asistencialista, los antecedentes mencionados y desarrollados en el texto revelan que la modalidad de trabajo de los gobiernos locales es variada. Si bien no renuncian al componente asistencial -área fundamental de su función-, la integran con acciones y decisiones relevantes en cuanto a organizar su labor de planificación, demostrando un gran dinamismo que los aleja significativamente del estereotipo de organismos pasivos y puramente asistencialistas. No se trata, sin duda, de una realidad consolidada ni de un fenómeno homogéneo, pero a partir del análisis realizado resulta claro que hay un conjunto importante de municipalidades que ha explorado y desarrollado una nueva manera de hacer política, con un enfoque más activo y una asunción de responsabilidad más directa en la promoción del desarrollo económico local.

Finalmente, cabe hacer hincapié en que el presente estudio podría constituir una herramienta analítica útil y de interés para los hacedores de la política pública local, quienes con escaza frecuencia tienen acceso a estudios económicos que profundicen en esta temática. Además, y en la perspectiva de futuras líneas de estudio, contar con una clasificación como la que aquí se presenta permitirá llevar a cabo investigaciones posteriores que incluyan un catastro de las políticas de desarrollo económico-productivo efectivamente implementadas, el cual se podría elaborar sobre la base de encuestas dirigidas a los agentes encargados del desarrollo económico local o del examen de la información contenida en la cuenta pública de la administración local que establece la ley (art. 67 de la Ley Orgánica Constitucional de Municipalidades). La evaluación del impacto real de cada una de estas políticas e iniciativas efectivamente desarrolladas es también fuente de posibles investigaciones.

\section{Bibliografía}

Alayón, N. R. (1991), Asistencia y asistencialismo: ¿pobres controlados o erradicación de la pobreza?, Río Piedras, Universidad de Puerto Rico.

Alburquerque, F. (2013), "Economía del desarrollo y desarrollo territorial" [en línea] http://www.conectadel. org/wp-content/uploads/downloads/2015/03/E\%C2\%AADesarrollo-y-Desarrollo-Territorial-3.01.pdf.

_(2004), "El enfoque del desarrollo económico local” [en línea] http://www.flacsoandes.edu.ec/sites/default/ files/agora/files/1251776298.area_enfoque_del_0.pdf.

Alburquerque, F. y M. Dini (2008), "Módulo 1: empresas, cadenas productivas y territorio. Introducción a los conceptos de innovación, productividad y competitividad" [en línea] http://www.conectadel.org/ wp-content/uploads/downloads/2013/02/modulo1resumenpdf2011-110623131205-phpapp02.pdf.

Arredondo, J., F. Toro y F. Olea (2007), Política pública y planificación participativa: una mirada desde el ámbito local, Santiago, Ministerio de Planificación y Cooperación (MIDEPLAN)/Secretaría Regional de Planificación y Coordinación (SERPLAC).

Artigas, C. (2005), "Una mirada a la protección social de los derechos humanos y otros contextos internacionales", serie Políticas Sociales, № 110 (LC/L.2354-P), Santiago, Comisión Económica para América Latina y el Caribe (CEPAL). 
Becattini, G. (2002), "Del distrito industrial marshalliano a la 'teoría del distrito' contemporánea: una breve reconstrucción crítica", Investigaciones Regionales, $N^{0} 1$ [en línea] https://old.aecr.org/ images//ImatgesArticles/2007/01-Becattini.pdf?_ga=2.249296007.1471150742.15389986412111654584.1538998641.

Bianchi, P. y S. Labory (2014), "The role of governance and government in the resilience of regions: the case of the 2012 earthquake in the Emilia-Romagna region in Italy" [en línea] https://halshs.archives-ouvertes. fr/halshs-01166138.

Bravo, S. M. (2012), "Implementación de la política de fomento en Chile: una aproximación a partir de la experiencia de las unidades de desarrollo económico a nivel municipal", Santiago, Universidad de Chile [en línea] http://repositorio.uchile.cl/bitstream/handle/2250/112502/cf-bravo_ss.pdf?sequence=1\&isAllowed=y.

Camuffo, A. y R. Grandinetti (2005), "I distretti industriali come economie della conoscenza", Argomenti, $N^{\circ} 15$ [en línea] https://www.francoangeli.it/riviste/Scheda_Rivista.aspx?IDArticolo=26026\&Tipo=Artic olo\%20PDF\&idRivista=100.

Garzón, A. y A. Guamán (coords.) (2015), El trabajo garantizado: una propuesta necesaria frente al desempleo y la precarización, Madrid, Akal.

Illanes, M. A. (2010), "La república de la gratitud: pérdida de democracia y 'chorreo' capitalista en Chile actual”, Ciencias, tecnologías, culturas. El desafío del conocimiento, C. Parker y F. Estenssoro (eds.), Santiago, Explora/Universidad de Santiago de Chile (USACH).

Montecinos, E. (2006), "Descentralización y democracia en Chile: análisis sobre la participación ciudadana en el presupuesto participativo y el plan de desarrollo comunal", Revista de Ciencia Política, vol. 26, № 2 , Santiago, Pontificia Universidad Católica de Chile.

Naciones Unidas (2016), "Proyecto de documento final de la Conferencia de las Naciones Unidas sobre la Vivienda y el Desarrollo Urbano Sostenible (Hábitat III)" (A/CONF.226/4), Nueva York, Asamblea General.

Olea, F. (2011), Los Planes de Desarrollo Comunal (Pladecos). Región Metropolitana de Santiago, Santiago, Secretaría Regional de Planificación y Coordinación (SERPLAC).

Orellana, A., J. A. Mena y M. Montes (2016), "Plan de desarrollo comunal: ¿el instrumento rector de la gestión municipal en Chile?", Revista INVI, vol. 31, № 87, Santiago, Universidad de Chile.

Ruz, M. Á. y otros (2014), "Planes de desarrollo comunal: propuestas para mejorar su efectividad como instrumento de planificación, participación y rendición de cuentas municipal”, Propuestas para Chile, I. Irarrázaval, C. Pozo y M. Letelier (eds.), Santiago, Pontificia Universidad Católica de Chile.

Salazar, G. (2013), "Una perspectiva social sobre la historia del municipio en Chile", Revista Iberoamericana de Estudios Municipales, año 4, № 7, Santiago, Universidad Autónoma de Chile.

Valenzuela, J. P. (2007), Análisis prospectivo de las capacidades institucionales y de gestión de los gobiernos subnacionales en Chile, Washington, D.C., Banco Interamericano de Desarrollo (BID).

Ziccardi, A. (coord.) (2004), Participación ciudadana y políticas sociales del ámbito local, Ciudad de México, Universidad Nacional Autónoma de México. 